\title{
MOVING GRIFFITH CRACK IN AN ORTHOTROPIC STRIP WITH PUNCHES AT BOUNDARY FACES
}

\author{
S. MUKHERJEE AND S. DAS
}

Received 20 May 2005

Integral transform technique is employed to solve the elastodynamic problem of steadystate propagation of a Griffith crack centrally situated along the midplane of orthotropic strip of finite thickness $2 h$ and subjected to point loading with centrally situated moving punches under constant pressure along the boundaries of the layer. The problem is reduced to the solution of a pair of simultaneous singular integral equations with Cauchytype singularities which have finally been solved through the finite Hilbert transform technique. For large $h$, analytical expression for the stress intensity factor at the crack tip is obtained. Graphical plots of the numerical results are also presented.

\section{Introduction}

Preexisting cracks in material structures interact to form major cracks leading to fracture. So the study of interaction of cracks in a medium under the action of external loads seems to be extremely important for the design and construction of safe material structures.

Several authors including Dhaliwal [3], Satapathy and Parhi [6], Cinar and Erdogan [1], Piva and Viola [5], Kassir and Tse [4], and Singh et al. [7] studied problems of Griffith cracks in orthotropic elastic materials. Recently, Das and Debnath [2] solved a problem for determining the stress intensity factors around the Griffith crack in a punched layer by reducing the problem to the solution of simultaneous integral equations, which are ultimately solved by using Tchebyshev polynomials.

In the present paper, the elastodynamic problem of the steady-state propagation of the Griffith crack in an orthotropic strip of finite thickness and subjected to point loading with punches on both of its boundary faces has been considered. As in the Yoffe model [9], it is assumed here that the crack is propagating with constant speed $c$ and without change in length along the positive $x$-axis. It is also assumed that the boundaries of the strip are subjected to central punches moving with the same velocity $c$. The problem is reduced to the solution of a pair of simultaneous singular integral equations with Cauchytype singularities. The integral equations are then solved using finite Hilbert transform technique. The expression for the stress intensity factor at the crack tip has been obtained 
for large values of $h$. Numerical calculations are carried out for the cases of $\alpha$-Uranium and graphical plots of the results are presented.

\section{Formulation of the problem}

Consider the plane elastodynamic problem of moving Griffith crack of finite length situated symmetrically in the midplane of an infinite orthotropic strip of thickness $2 h$ with central punch at the upper face. Let the coordinate axes $X, Y$, and $Z$ coincide with the axes of elastic symmetry of the material. As in the Yoffe model, it is assumed that the crack is defined by the relations $|X| \leq a, Y= \pm 0$ is opened by internal normal traction $p_{1}(x)$ and the punch is defined by $|X| \leq a, Y= \pm h$ is subjected to normal pressure $p_{2}$ and these are propagating without change in length with constant speed $c$ along the positive $X$-axis.

As the problem considered here is restricted to motion in the $X-Y$ plane, in the absence of body forces, the equations of motion are

$$
\begin{aligned}
& C_{11} \frac{\partial^{2} U}{\partial X^{2}}+C_{66} \frac{\partial^{2} U}{\partial Y^{2}}+\left(C_{12}+C_{66}\right) \frac{\partial^{2} V}{\partial X \partial Y}=\rho \frac{\partial^{2} U}{\partial t^{2}} \\
& C_{22} \frac{\partial^{2} V}{\partial Y^{2}}+C_{66} \frac{\partial^{2} V}{\partial X^{2}}+\left(C_{12}+C_{66}\right) \frac{\partial^{2} U}{\partial X \partial Y}=\rho \frac{\partial^{2} V}{\partial t^{2}}
\end{aligned}
$$

in which $U=U(X, Y, t), V=V(X, Y, t)$ are the displacement components in the $X$ and $Y$ directions, $t$ is the time, $\rho$ is the density of the material, and $C_{i j}$ 's are the elastic constants. The stress-displacement relations are

$$
\begin{aligned}
\sigma_{x x} & =C_{11} \frac{\partial U}{\partial X}+C_{12} \frac{\partial V}{\partial Y}, \\
\sigma_{y y} & =C_{12} \frac{\partial U}{\partial X}+C_{22} \frac{\partial V}{\partial Y}, \\
\sigma_{x y} & =C_{66}\left(\frac{\partial U}{\partial Y}+\frac{\partial V}{\partial X}\right) .
\end{aligned}
$$

Using Galilean transformation, $x=X-c t, y=Y, t=t$, and assuming that $U=u(x, y)$, $V=v(x, y),(2.1)$ becomes independent of $t$ and reduces to

$$
\begin{aligned}
& \left(C_{11}-c^{2} \rho\right) \frac{\partial^{2} u}{\partial x^{2}}+C_{66} \frac{\partial^{2} u}{\partial y^{2}}+\left(C_{12}+C_{66}\right) \frac{\partial^{2} v}{\partial x \partial y}=0 \\
& C_{22} \frac{\partial^{2} v}{\partial y^{2}}+\left(C_{66}-c^{2} \rho\right) \frac{\partial^{2} v}{\partial x^{2}}+\left(C_{12}+C_{66}\right) \frac{\partial^{2} u}{\partial x \partial y}=0
\end{aligned}
$$

The Mach numbers $M_{j}=c / v_{j}(j=1,2)$, in which $v_{1}=\left(C_{11} / \rho\right)^{1 / 2}$ is called the velocity of dilatational wave and $v_{2}=\left(C_{66} / \rho\right)^{1 / 2}$ is called the velocity of shear wave, are assumed to be less than 1 for a subsonic propagation. 
As the problem under discussion is symmetric with respect to $x$-axis, it is sufficient to consider the half strip $0 \leq y \leq h$. Therefore, the boundary conditions of the problems are

$$
\begin{aligned}
\sigma_{y y}(x, h) & =-p_{2}, \quad|x| \leq a, \\
v(x, h) & =0, \quad|x|>a, \\
\sigma_{x y}(x, h) & =0, \quad|x|<\infty, \\
\sigma_{y y}(x, 0) & =-p_{1}(x), \quad|x| \leq a, \\
v(x, 0) & =0, \quad|x|>a, \\
\sigma_{x y}(x, 0) & =0, \quad|x|<\infty .
\end{aligned}
$$

In addition, all the components of stress and displacement vanish at the remote distances from the crack.

\section{Solution of the problem}

An appropriate integral solution to (2.3) can be written as

$$
\begin{aligned}
& u(x, y)=\int_{0}^{\infty} A(s, y) \sin s x d s, \\
& v(x, y)=\int_{0}^{\infty} B(s, y) \cos s x d s,
\end{aligned}
$$

where $A(s, y)$ and $B(s, y)$ are arbitrary functions. Substituting (3.1) into (2.3), the following relations are found to govern $A(s, y)$ and $B(s, y)$ :

$$
\begin{aligned}
& \left(C_{11}-c^{2} \rho\right) s^{2} A-C_{66} \frac{d^{2} A}{d y^{2}}+\left(C_{12}+C_{66}\right) s \frac{d B}{d y}=0 \\
& \left(C_{66}-c^{2} \rho\right) s^{2} B-C_{22} \frac{d^{2} B}{d y^{2}}-\left(C_{12}+C_{66}\right) s \frac{d A}{d y}=0 .
\end{aligned}
$$

These equations have the solutions

$$
\begin{aligned}
& A(s, y)=A_{1}(s) \cosh \left(\gamma_{1} s y\right)+A_{2}(s) \cosh \left(\gamma_{2} s y\right)+C_{1}(s) \sinh \left(\gamma_{1} s y\right)+C_{2}(s) \sinh \left(\gamma_{2} s y\right) \\
& B(s, y)=B_{1}(s) \sinh \left(\gamma_{1} s y\right)+B_{2}(s) \sinh \left(\gamma_{2} s y\right)+D_{1}(s) \cosh \left(\gamma_{1} s y\right)+D_{2}(s) \cosh \left(\gamma_{2} s y\right),
\end{aligned}
$$

where $\gamma_{1}$ and $\gamma_{2}$ are the positive roots of the equation

$$
C_{22} C_{66} \gamma^{4}+\left[\left(C_{12}+C_{66}\right)^{2}-C_{22}\left(C_{11}-c^{2} \rho\right)-C_{66}\left(C_{66}-c^{2} \rho\right)\right] \gamma^{2}+\left(C_{11}-c^{2} \rho\right)\left(C_{66}-c^{2} \rho\right)=0 .
$$


3160 Moving Griffith crack in an orthotropic strip

In (3.3), $A_{j}(s)$ and $C_{j}(s)(j=1,2)$ are arbitrary functions and $B_{j}(s), D_{j}(s)$ are related to $A_{j}(s), C_{j}(s)$ by the relations

$$
B_{j}(s)=-\frac{\alpha_{j}}{\gamma_{j}} A_{j}(s), \quad D_{j}(s)=-\frac{\alpha_{j}}{\gamma_{j}} C_{j}(s),
$$

where

$$
\alpha_{j}=\frac{C_{11}-c^{2} \rho-\gamma_{j}^{2} C_{66}}{C_{12}+C_{66}} \quad(j=1,2) .
$$

The expressions for the stress are

$$
\begin{aligned}
\sigma_{x x}(x, y)=\int_{0}^{\infty}[ & \left(C_{11}-\alpha_{1} C_{12}\right) A_{1}(s) \cosh \left(\gamma_{1} s y\right)+\left(C_{11}-\alpha_{2} C_{12}\right) A_{2}(s) \cosh \left(\gamma_{2} s y\right) \\
& \left.+\left(C_{11}-\alpha_{1} C_{12}\right) C_{1}(s) \sinh \left(\gamma_{1} s y\right)+\left(C_{11}-\alpha_{2} C_{12}\right) C_{2}(s) \sinh \left(\gamma_{2} s y\right)\right] s \cos s x d s
\end{aligned}
$$

$$
\begin{array}{r}
\sigma_{y y}(x, y)=\int_{0}^{\infty}\left[\left(C_{12}-\alpha_{1} C_{22}\right) A_{1}(s) \cosh \left(\gamma_{1} s y\right)+\left(C_{12}-\alpha_{2} C_{22}\right) A_{2}(s) \cosh \left(\gamma_{2} s y\right)\right. \\
\left.+\left(C_{12}-\alpha_{1} C_{22}\right) C_{1}(s) \sinh \left(\gamma_{1} s y\right)+\left(C_{12}-\alpha_{2} C_{12}\right) C_{2}(s) \sinh \left(\gamma_{2} s y\right)\right] s \cos s x d s,
\end{array}
$$

$$
\begin{aligned}
\sigma_{x y}(x, y)=C_{66} \int_{0}^{\infty} & \frac{\beta_{1}}{\gamma_{1}} A_{1}(s) \sinh \left(\gamma_{1} s y\right)+\frac{\beta_{2}}{\gamma_{2}} A_{2}(s) \sinh \left(\gamma_{2} s y\right)+\frac{\beta_{1}}{\gamma_{1}} C_{1}(s) \cosh \left(\gamma_{1} s y\right) \\
& \left.+\frac{\beta_{2}}{\gamma_{2}} C_{2}(s) \cosh \left(\gamma_{2} s y\right)\right] s \sin s x d s,
\end{aligned}
$$

where

$$
\beta_{j}=\alpha_{j}+\gamma_{j}^{2} \quad(j=1,2)
$$

applying (2.9), we get

$$
C_{2}(s)=-\frac{\mu_{1}}{\mu_{2}} C_{1}(s)
$$

where

$$
\mu_{j}=C_{66} \frac{\beta_{j}}{\gamma_{j}} .
$$

Boundary condition (2.8) with (3.1) and (3.11) gives

$$
\int_{0}^{\infty} C_{1}(s) \cos s x d s=0, \quad|x|>a .
$$


Boundary condition (2.5) with (3.1) and (3.11) gives

$$
\begin{aligned}
\int_{0}^{\infty} & {\left[\frac{\alpha_{1}}{\gamma_{1}} A_{1}(s) \sinh \left(\gamma_{1} s h\right)+\frac{\alpha_{2}}{\gamma_{2}} A_{2}(s) \sinh \left(\gamma_{2} s h\right)\right.} \\
& \left.+C_{1}(s)\left\{\frac{\alpha_{1}}{\gamma_{1}} \cosh \left(\gamma_{1} s h\right)-\frac{\mu_{1}}{\mu_{2}} \frac{\alpha_{2}}{\gamma_{2}} \cosh \left(\gamma_{2} s h\right)\right\}\right] \cos s x d s=0, \quad|x|>a .
\end{aligned}
$$

Setting

$$
\begin{gathered}
C_{1}(s)=\frac{1}{s} \int_{0}^{a} f_{1}(t) \sin s t d t \\
\frac{\alpha_{1}}{\gamma_{1}} A_{1}(s) \sinh \left(\gamma_{1} s h\right)+\frac{\alpha_{2}}{\gamma_{2}} A_{2}(s) \sinh \left(\gamma_{2} s h\right) \\
+C_{1}(s)\left\{\frac{\alpha_{1}}{\gamma_{1}} \cosh \left(\gamma_{1} s h\right)-\frac{\mu_{1}}{\mu_{2}} \frac{\alpha_{2}}{\gamma_{2}} \cosh \left(\gamma_{2} s h\right)\right\}=\frac{1}{s} \int_{0}^{a} f_{2}(t) \sin s t d t
\end{gathered}
$$

where $f_{1}(t)$ and $f_{2}(t)$ are unknown functions of $t$ and using the result

$$
\int_{0}^{\infty} \frac{\sin s t \cdot \cos s x}{s} d s= \begin{cases}\frac{\pi}{2}, & t>x \\ 0, & t<x\end{cases}
$$

(3.15) is identically satisfied.

Using (3.15), the boundary condition (2.6) leads to

$$
\begin{aligned}
& A_{1}(s)=-\frac{\left[1+\delta_{1}(s)\right]}{s} \int_{0}^{a} f_{1}(t) \sin s t d t+\frac{\delta_{2}(s)}{s} \int_{0}^{a} f_{2}(t) \sin s t d t \\
& A_{2}(s)=\frac{\mu_{1}}{\mu_{2} s}\left[1+\delta_{3}(s)\right] \int_{0}^{a} f_{1}(t) \sin s t d t-\frac{\delta_{4}(s)}{s} \int_{0}^{a} f_{2}(t) \sin s t d t
\end{aligned}
$$

where

$$
\begin{aligned}
& \delta_{1}(s)=\frac{e^{-\gamma_{1} s h}}{\sinh \left(\gamma_{1} s h\right)}, \\
& \delta_{2}(s)=\frac{D_{1}}{D_{2} \sinh \left(\gamma_{1} s h\right)}, \\
& \delta_{3}(s)=\frac{e^{-\gamma_{2} s h}}{\sinh \left(\gamma_{2} s h\right)}, \\
& \delta_{4}(s)=\frac{\mu_{1}}{\mu_{2}} \cdot \frac{D_{1}}{D_{2}} \cdot \frac{1}{\sinh \left(\gamma_{2} s h\right)} .
\end{aligned}
$$


3162 Moving Griffith crack in an orthotropic strip

Finally, the boundary conditions (2.4) and (2.7), in conjunction with (3.8), lead to the following integral equations:

$$
\begin{aligned}
& \int_{0}^{a} \frac{t f_{1}(t) d t}{t^{2}-x^{2}}+\frac{1}{2} \int_{0}^{a} K_{11}(x, t) f_{1}(t) d t+\frac{1}{2} \int_{0}^{a} K_{12}(x, t) f_{2}(t) d t=-D_{1} p_{1}(x), \quad|x| \leq a, \\
& \int_{0}^{a} \frac{t f_{2}(t) d t}{t^{2}-x^{2}}+\frac{1}{2} \int_{0}^{a} K_{21}(x, t) f_{1}(t) d t+\frac{1}{2} \int_{0}^{a} K_{22}(x, t) f_{2}(t) d t=D_{2} p_{2}, \quad|x| \leq a,
\end{aligned}
$$

where

$$
\begin{aligned}
K_{i j}(x, t)=\int_{0}^{\infty} d_{i j}(s)\{\sin s(t+x)+\sin s(t-x)\} d s \quad(i, j=1,2) \\
d_{11}(s)=d_{22}(s)=\frac{1}{D^{\prime}}\left[\mu_{1} \eta_{2} \delta_{3}(s)-\mu_{2} \eta_{1} \delta_{1}(s)\right], \\
d_{12}(s)=\frac{\mu_{2}}{D^{\prime}}\left[\eta_{1} \delta_{2}(s)-\eta_{2} \delta_{4}(s)\right], \\
d_{21}(s)=D_{2}\left[\delta_{5}(s)-\frac{\mu_{1}}{\mu_{2}} \delta_{6}(s)\right], \\
\delta_{5}(s)=\eta_{1}\left\lfloor e^{-\gamma_{1} s h}+\delta_{1}(s) \cosh \left(\gamma_{1} s h\right)\right\rfloor, \\
\delta_{6}(s)=\eta_{2}\left\lfloor e^{-\gamma_{2} s h}+\delta_{3}(s) \cosh \left(\gamma_{2} s h\right)\right\rfloor,
\end{aligned}
$$

with

$$
\begin{gathered}
D_{1}=\frac{\mu_{2}}{D^{\prime}}, \quad D_{2}=\frac{1}{D^{\prime}}\left(\frac{\alpha_{1} \mu_{2}}{\gamma_{1}}-\frac{\alpha_{2} \mu_{1}}{\gamma_{2}}\right), \\
D^{\prime}=\mu_{1} \eta_{2}-\mu_{2} \eta_{1} \\
\eta_{j}=C_{12}-\alpha_{j} C_{22} \quad(j=1,2) .
\end{gathered}
$$

Using estimates of $\delta_{i}(s)(i=1,2, \ldots, 6)$ for large $h,(3.20)$ leads to

$$
\begin{aligned}
& K_{11}(x, t)=-\frac{2 \eta_{1} \mu_{2}}{D^{\prime}} L_{1}(x, t)+\frac{2 \mu_{1} \eta_{2}}{D^{\prime}} L_{2}(x, t)=K_{22}(x, t), \\
& K_{12}(x, t)=\frac{2 \mu_{2}^{2} \eta_{1}}{D_{2} D^{\prime 2}} N_{1}(x, t)-\frac{2 \mu_{1} \mu_{2} \eta_{2}}{D_{2} D^{\prime 2}} N_{2}(x, t), \\
& K_{21}(x, t)=2 \eta_{1} D_{2} N_{1}(x, t)-\frac{2 D_{1} \mu_{1} \eta_{2}}{\mu_{2}} N_{2}(x, t)
\end{aligned}
$$


where

$$
\begin{aligned}
L_{j}(x, t)= & \frac{t+x}{4 \gamma_{j}^{2} h^{2}+(t+x)^{2}}+\frac{t-x}{4 \gamma_{j}^{2} h^{2}+(t-x)^{2}} \\
& +\frac{t+x}{16 \gamma_{j}^{2} h^{2}+(t+x)^{2}}+\frac{t-x}{16 \gamma_{j}^{2} h^{2}+(t-x)^{2}}+\cdots, \\
N_{j}(x, t)= & \frac{t+x}{\gamma_{j}^{2} h^{2}+(t+x)^{2}}+\frac{t-x}{\gamma_{j}^{2} h^{2}+(t-x)^{2}} \\
& +\frac{t+x}{9 \gamma_{j}^{2} h^{2}+(t+x)^{2}}+\frac{t-x}{9 \gamma_{j}^{2} h^{2}+(t-x)^{2}}+\cdots \quad(j=1,2) .
\end{aligned}
$$

Now, expanding $L_{j}(x, t)$ and $N_{j}(x, t)(j=1,2)$ in powers of $1 / h$, for large $h$, we can get

$$
\begin{aligned}
& K_{11}(x, t)=K_{22}(x, t)=-\frac{\pi^{2} D_{3}}{6 h^{2}} \cdot t, \\
& K_{12}(x, t)=-\frac{\pi^{2} \mu_{2} D_{3}}{2 h^{2} D_{2} D^{\prime 2}} \cdot t \\
& K_{21}(x, t)=\frac{\pi^{2} D_{2} D_{3} D^{\prime}}{2 \mu_{2} h^{2}} \cdot t
\end{aligned}
$$

Let us now consider the expansion of $f_{i}(t)(i=1,2)$ in the form

$$
f_{i}(t)=f_{i}^{(0)}(t)+\frac{1}{h^{2}} f_{i}^{(1)}(t)+O\left(\frac{1}{h^{4}}\right), \quad i=1,2,
$$

comparing coefficients of like powers of $1 / h,(3.19)$, under (3.25) and (3.26) immediately leads to the integral equations

$$
\begin{aligned}
& \int_{0}^{a} \frac{2 t f_{1}^{(0)}(t) d t}{t^{2}-x^{2}}=-2 D_{1} p_{1}(x), \\
& \int_{0}^{a} \frac{2 t f_{2}^{(0)}(t) d t}{t^{2}-x^{2}}=2 D_{2} p_{2} \\
& \int_{0}^{a} \frac{2 t f_{1}^{(1)}(t) d t}{t^{2}-x^{2}}=\frac{\pi^{2} D_{3}}{6} \int_{0}^{a} t f_{1}^{(0)}(t) d t-\frac{\pi^{2} \mu_{2} D_{3}}{2 D_{2} D^{\prime 2}} \int_{0}^{a} t f_{2}^{(0)}(t) d t \\
& \int_{0}^{a} \frac{2 t f_{2}^{(1)}(t) d t}{t^{2}-x^{2}}=\frac{\pi^{2} D_{3}}{6} \int_{0}^{a} t f_{2}^{(0)}(t) d t-\frac{\pi^{2}}{2} \frac{D_{2} D_{3} D^{\prime}}{\mu_{2}} \int_{0}^{a} t f_{1}^{(0)}(t) d t
\end{aligned}
$$

where

$$
D_{3}=\frac{1}{D^{\prime}}\left(\frac{\mu_{2} \eta_{1}}{\gamma_{1}^{2}}-\frac{\mu_{1} \eta_{2}}{\gamma_{2}^{2}}\right) .
$$


3164 Moving Griffith crack in an orthotropic strip

Using Tricomi result (cf. Tricomi [8]) and employing

$$
p_{1}(x)=p_{1} \delta\left(x-x_{0}\right),
$$

where $\delta(\cdot)$ is the Dirac delta function, we have the following results:

$$
\begin{aligned}
& f_{1}^{(0)}(t)=\frac{4 D_{1} p_{1}}{\pi^{2}} \cdot \frac{x_{0}^{2} \sqrt{a^{2}-x_{0}{ }^{2}}}{t \sqrt{a^{2}-t^{2}}\left(x_{0}{ }^{2}-t^{2}\right)}+\frac{2 D_{1} p_{1}}{\pi t \sqrt{a^{2}-t^{2}}}, \\
& f_{2}{ }^{(0)}(t)=\frac{2 D_{2} p_{2}}{\pi} \cdot \frac{t}{\sqrt{a^{2}-t^{2}}}, \\
& f_{1}^{(1)}(t)=\frac{m t}{\pi \sqrt{a^{2}-t^{2}}}, \\
& f_{2}{ }^{(1)}(t)=\frac{n t}{\pi \sqrt{a^{2}-t^{2}}},
\end{aligned}
$$

where

$$
\begin{aligned}
& m=\frac{\pi^{2} D_{1} D_{3} p_{1}}{6}-\frac{\pi^{2} D_{1} D_{3} p_{2} a^{2}}{4 D^{\prime}} \\
& n=\frac{\pi^{2} D_{2} D_{3} p_{2} a^{2}}{12}-\frac{\pi^{2} D_{2} D_{3} p_{1}}{2} .
\end{aligned}
$$

Using (3.30), (3.26) yields

$$
\begin{aligned}
& f_{1}(t)=\frac{4 D_{1} p_{1}}{\pi^{2}} \cdot \frac{x_{0}^{2} \sqrt{a^{2}-x_{0}^{2}}}{t \sqrt{a^{2}-t^{2}}\left(x_{0}^{2}-t^{2}\right)}+\frac{2 D_{1} p_{1}}{\pi t \sqrt{a^{2}-t^{2}}}+\frac{1}{h^{2}}\left(\frac{p_{1}}{6}-\frac{p_{2} a^{2}}{4 D^{\prime}}\right) \frac{\pi D_{1} D_{3} t}{\sqrt{a^{2}-t^{2}}}+O\left(\frac{1}{h^{4}}\right), \\
& f_{2}(t)=\frac{2 D_{2} p_{2}}{\pi} \cdot \frac{t}{\sqrt{a^{2}-t^{2}}}+\frac{1}{h^{2}}\left(\frac{p_{2} a^{2}}{6}-p_{1}\right) \frac{\pi D_{2} D_{3} t}{2 \sqrt{a^{2}-t^{2}}}+O\left(\frac{1}{h^{4}}\right) .
\end{aligned}
$$

The stress intensity factor at the crack tip $x=a$ is

$$
K_{I}=\lim _{x \rightarrow a+} \sqrt{2(x-a)} \sigma_{y y}(x, 0),
$$

and is calculated as

$$
K_{I}=\left(\frac{2 D_{1} p_{1}}{\pi} \cdot \frac{1}{a \sqrt{a}} \cdot \frac{x_{0}^{2}}{\sqrt{a^{2}-x_{0}^{2}}}\right)-\frac{D_{1} p_{1}}{a^{3 / 2}}-\frac{1}{h^{2}}\left(\frac{p_{1}}{6}-\frac{p_{2} a^{2}}{4 D^{\prime}}\right)\left(\frac{\pi^{2} D_{1} D_{3}}{2} \sqrt{a}\right) .
$$

The stress component outside the crack region is calculated as

$$
\begin{aligned}
\sigma_{y y}(x, 0)= & \frac{2 p_{1}}{\pi} x_{0}^{2} \sqrt{a^{2}-x_{0}^{2}} \cdot \frac{1}{x \sqrt{x^{2}-a^{2}}\left(x^{2}-x_{0}^{2}\right)}-\frac{p_{1}}{x \sqrt{a^{2}-c^{2}}} \\
& +\frac{1}{h^{2}}\left(\frac{p_{1}}{6}-\frac{p_{2} a^{2}}{4 D^{\prime}}\right) \cdot \frac{\pi^{2} D_{3}}{2}\left(1-\frac{x}{\sqrt{x^{2}-a^{2}}}\right)+\frac{1}{h^{2}}\left(\frac{\pi^{2} D_{3} p_{2}}{2 D^{\prime}} a^{2}-\frac{\pi^{2} D_{3} p_{1}}{12}\right) .
\end{aligned}
$$


Table 4.1

\begin{tabular}{cccccc}
\hline & $C_{11}$ & $C_{22}$ & $C_{66}$ & $C_{12}$ & $\rho$ \\
\hline$\alpha$-Uranium & 21.47 & 19.36 & 7.43 & 4.65 & 19.07 \\
\hline
\end{tabular}

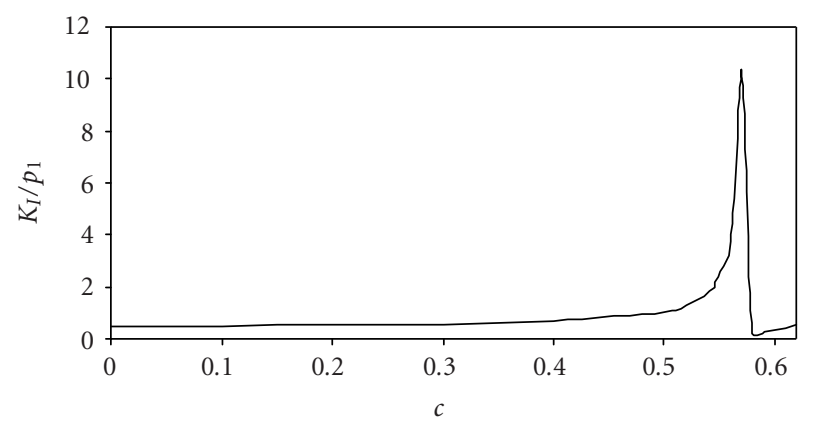

Figure 4.1 Plot of $K_{I} / p_{1}$ versus $c$ for $h=6, x_{0} / a=0.6, p_{2} / p_{1}=1.5$.

\section{Numerical results and discussion}

As a particular case, we have considered the orthotropic strip to be made of $\alpha$-Uranium with material constants as given in Table 4.1.

The normalized stress intensity factor at the crack tip $x=a$ has been plotted for different subsonic propagation of the crack as well as the punches and for different depth of the half-strip maintaining always half-crack length $a=0.5$.

Figure 4.1 shows the plotting of the normalized stress intensity factor when the depth of the half-strip is 6 units and the punch pressure is 1.5 times the point load applied on the crack face at a distance of 3/5th of half-crack length from the center of the crack. The figure shows an amplification in the stress intensity factor which changes negligibly up to 4 units with change in the crack velocity $c$ and then starts increasing quickly and jumps abruptly near $c=0.5736$ unit and then starts oscillating, which is expected as there is a change in propagation phase from subsonic to supersonic.

Figure 4.2 plots the normalized stress intensity factor against the subsonic velocity for different positions of the point load along the crack length. In all these plottings, the halfstrip depth is maintained at $h=4$ units and the punch pressure is maintained at half the point load. It is observed that the further the point load from the crack tip, the larger the amplification and the oscillations in all the cases starting at the same value of $c$, that is, for $c=0.5736$.

Figure 4.3 shows the plottings of the normalized stress intensity factor against the ratio $p_{2} / p_{1}$ for $c=0.5736$, where the stress intensity factor shoots up abruptly. The plottings are obtained for different half-strip depths. It is found that when the depth is large, the increase in the punch pressure almost does not affect the stress intensity factor at the crack tip. As the depth decreases, the effect of increase in the punch pressure is noticed on the plotting of the stress intensity factor. 


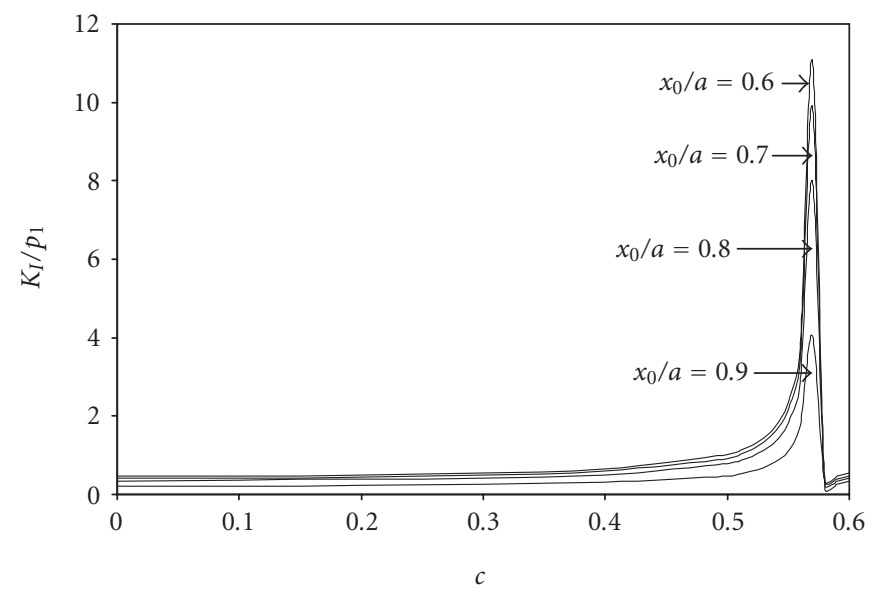

Figure 4.2 Plot of $K_{I} / p_{1}$ versus $c$ for different $x_{0} / a, h=4, p_{2} / p_{1}=0.5$.

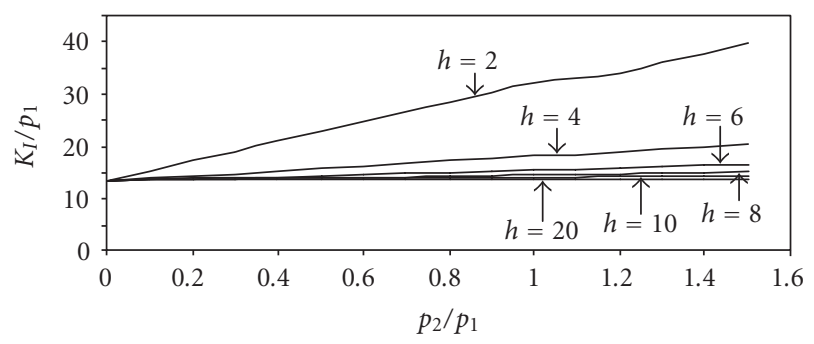

Figure 4.3 Plot of $K_{I} / p_{1}$ versus $p_{2} / p_{1}$ at $x_{0} / a=0.6, c=0.5736$.

Stress intensity factor increases steadily with increase in the punch pressure. It is also noticed that the rate of increase in the stress intensity factor with increase in punch pressure decreases at the point where the value of the punch pressure crosses the value of the point load from low to high.

\section{References}

[1] A. Cinar and F. Erdogan, The crack and wedging problem for an orthotropic strip, Internat. J. Fracture 23 (1983), 83-102.

[2] S. Das and L. Debnath, Interaction between Griffith cracks in a sandwiched orthotropic layer, Appl. Math. Lett. 16 (2003), no. 4, 609-617.

[3] R. S. Dhaliwal, Two coplanar cracks in an infinitely long orthotropic elastic strip, Utilitas Math. 4 (1973), 115-128.

[4] M. K. Kassir and S. Tse, Moving Griffith crack in an orthotropic material, Int. J. Engng. Sci. 21 (1983), 315-325.

[5] A. Piva and E. Viola, Crack propagating in an orthotropic medium, Engrg. Fracture Mech. 29 (1988), 535-548.

[6] P. K. Satapathy and H. Parhi, Stresses in an orthotropic strip containing a Griffith crack, Int. J. Engng. Sci. 16 (1978), 147-154. 
[7] P. C. Singh, B. Patra, and T. N. Maulik, Two collinear Griffith cracks in an orthotropic strip with punches, Appl. Mech. Eng. 3 (1998), no. 4, 621-653.

[8] F. G. Tricomi, On the finite Hilbert transformation, Quart. J. Math. Oxford Ser. (2) 2 (1951), 199-211.

[9] E. H. Yoffe, The moving Griffith crack, Philos. Mag. (7) 42 (1951), 739-750.

S. Mukherjee: Department of Mathematics, B. P. Poddar Institute of Management \& Technology, Poddar Vihar, 137 VIP Road, Kolkata-700052, West Bengal, India

E-mail address: subir_mukh123@hotmail.com

S. Das: Department of Mathematics, B. P. Poddar Institute of Management \& Technology, Poddar Vihar, 137 VIP Road, Kolkata-700052, West Bengal, India

E-mail address: sananda_das@hotmail.com 


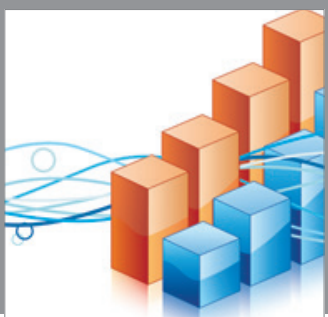

Advances in

Operations Research

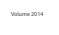

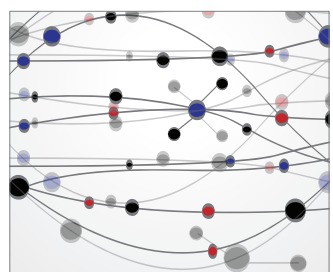

\section{The Scientific} World Journal
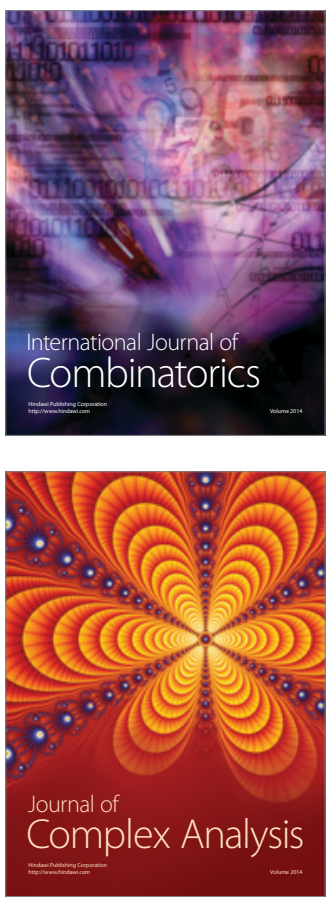

International Journal of

Mathematics and

Mathematical

Sciences
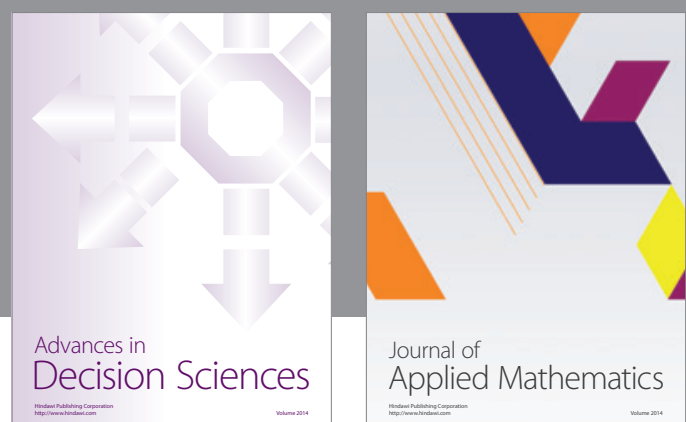

Journal of

Applied Mathematics
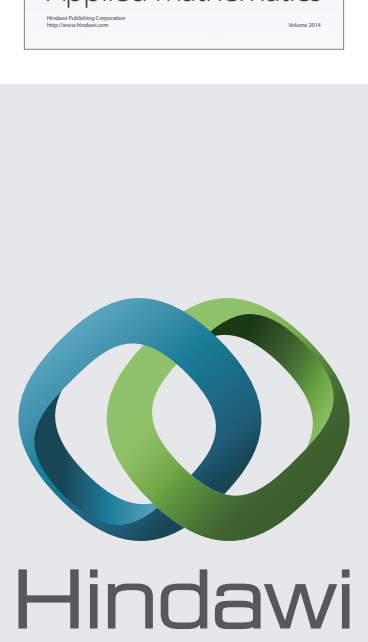

Submit your manuscripts at http://www.hindawi.com
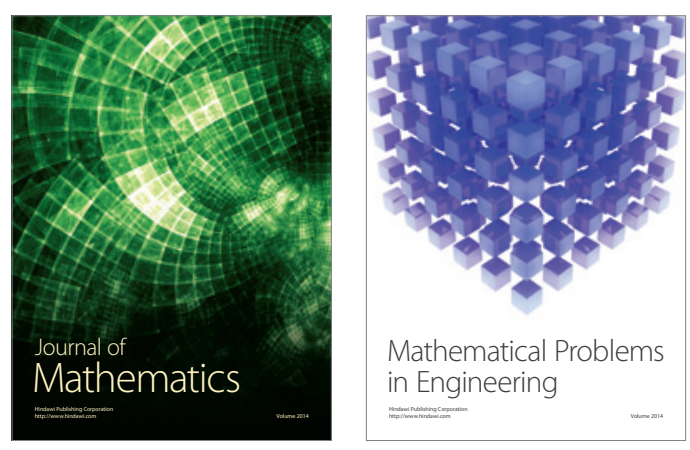

Mathematical Problems in Engineering
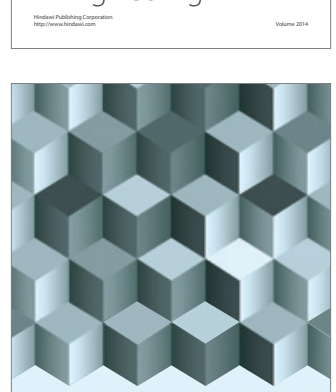

Journal of

Function Spaces
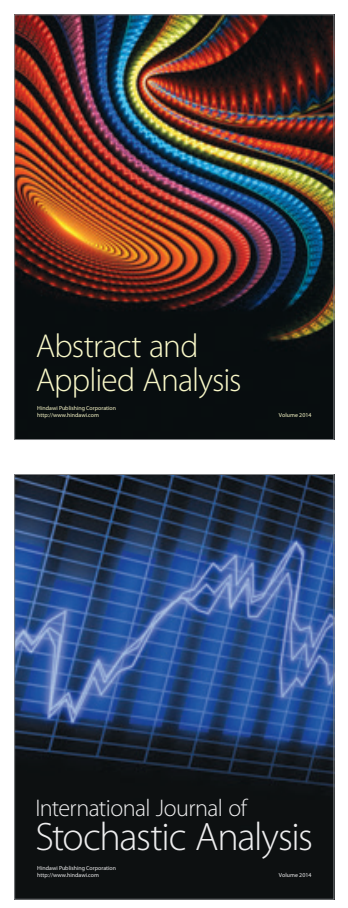

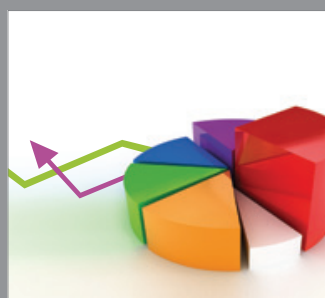

ournal of

Probability and Statistics

Promensencen
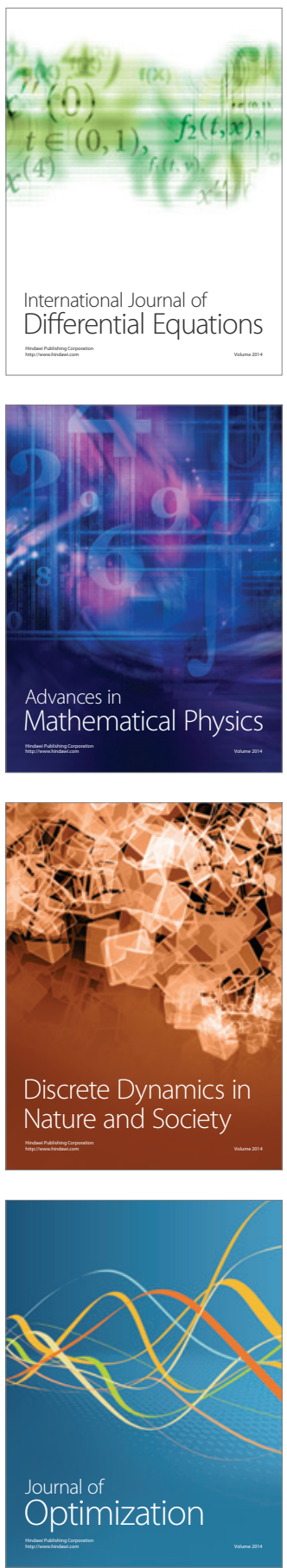